\title{
Zwitserland
}

\section{Länderbericht Schweiz}

Prof. Dr. oec. Hans Rainer Künzle*

\section{Stellung des überlebenden Ehegatten}

Das eheliche Güterrecht (Art. 181-251 Zivilgesetzbuch (ZGB)) befasst sich mit dem Vermögensrecht und zwar sowohl im Verhältnis zwischen den Ehegatten, als auch der Ehegatten zu Dritten. ${ }^{1}$

\subsection{Güterrechtliche Auseinandersetzung}

Seit dem 1. Januar 1988 kennt das schweizerische Eherecht die drei Güterstände der Errungenschaftsbeteiligung (Art. 196-220 ZGB), der Gütergemeinschaft (Art. 221-246 ZGB) und der Gütertrennung (Art. 247-251 ZGB). Die Ehegatten können unter diesen Güterständen ziemlich frei ${ }^{2}$ (durch Ehevertrag) wählen. ${ }^{3}$

* Prof. Dr. Hans Rainer Künzle ist Rechtsanwalt, Titularprofessor für Privatrecht und Privatrechtsvergleichung an der Universität Zürich und Of counsel von Kendris AG in Zürich.

1. Vgl. Alexandra Jungo, Kommentar zu Art. 181-251 ZGB, in: Kommentar zum Schweizer Privatrecht, hrsg. v. Peter Breitschmid und Alexandra Jungo, 3. Aufl., Zürich/Basel/Genf 2016 (zit. CHK-Jungo), Art. 181 ZGB N 1.

2. CHK-Jungo (Fn. 1), Art. 181 ZGB N 3: Ausnahmen findet man etwa in Art. 185, 187 und 189 ZGB (gerichtliche Anordnung der Gütertrennung bei Überschuldung etc.).

3. Der Ehevertrag ist notariell zu beurkunden (Art. 184 ZGB); von dieser Formvorschrift sind allerdings verschiedene Vorgänge befreit, wie etwa die Errichtung eines Inventars nach Art. 195a ZGB, die Zustimmung zu einer Verfügung nach Art. 201 ZGB oder der Verzicht auf eine Mehrwertbeteiligung nach Art. 206 ZGB, vgl. Heinz Hausheer/Regina AebiMüller, Kommentar zu Art. 181-251 ZGB, in: Basler Kommentar, Zivilgesetzbuch I (Art. 1-456 ZGB), hrsg. v. Heinrich Honsell etc. (zit. BSK ZGB I-Hausheer/Aebi-Müller), 4. Aufl., Basel 2010, Art. 182 ZGB N 5.

4. Weiter gehören persönliche Gegenstände, Genugtuungsansprüche Ersatzanschaffungen usw. zum Eigengut, vgl. BSK ZGB-I-Hausheer/ Aebi-Müller (Fn. 4), Art. 198 ZGB N 5 ff.

5. Dazu gehören auch Versicherungsleistungen als Ersatz für den Arbeitserwerb (AHV, BVG, 3. Säule, IV, ALV, Krankentaggeld), vgl. Heinz Hausheer/Ruth Reusser/Thomas Geiser, Kommentar zum schweizerischen Privatrecht, Band II: Das Familienrecht, 1. Abteilung: Das Eherecht, 3. Teilband: Das Güterrecht der Ehegatten, 1. Unterteilband: Allgemeine Vorschriften. Art. 181-195a ZGB - Der ordentliche Güterstand der Errungenschaftsbeteiligung, Art. 196-220 ZGB, Bern 1992, 2. Unterteilband: Die Gütergemeinschaft (Art. 221-246 ZGB) - Die Gütertrennung (Art. 247-251 ZGB), Bern 1996 (zit. BK-Hausheer/Reusser/ Geiser), Art. 197 ZGB N $50 \mathrm{ff}$.

6. CHK-Jungo (Fn. 1), Art. 197 ZGB N 6 ff.

7. Eine Ausnahme sieht Art. 199 ZGB vor: Zur Berufsausübung oder zum Gewerbebetrieb gehörende Errungenschaft wird als Eigengut erklärt.

8. Die Zuordnung zu den Massen spielt schon während der Ehe eine Rolle bei Fragen wie: Mehr- und Minderwertbeteiligungen (Art. 206 und 209 Abs. 2 ZGB), Ersatzanschaffungen (Art. 197 Abs. 2 Ziff. 5 und 198 Ziff. 4 ZGB), Hinzurechnungen (Art. 208 ZGB), Massenumteilungen (Art. 199 ZGB) und bei der Miteigentumsvermutung (Art. 200 Abs. 2 ZGB), vgl. CHK-Jungo (Fn. 1), Art. 196 ZGB N 1.

9. Den Fall der Scheidung kann man mit dem Ehevertrag nur unvollständig abdecken, vgl. CHK-Jungo (Fn. 1), Art. 181 ZGB N 7. 


\begin{tabular}{|c|c|c|c|}
\hline \multicolumn{4}{|c|}{ Errungenschaftsbeteiligung } \\
\hline Bilgengent. & Errungenschaft & Errungenschaft & Eiligengut \\
\hline Fintul & Frau & Mann & Mann \\
\hline
\end{tabular}

\subsubsection{Gütergemeinschaft}

Die Gütergemeinschaft war schon im kantonalen Recht stark verbreitet und wird vor allem dann benützt, wenn man die Hebelwirkung (Zuweisung des Gesamtgutes) verstärken, insbesondere den überlebenden Ehegatten (im Rahmen einer Erbschaftsplanung) besserstellen, will. ${ }^{10}$

\begin{tabular}{|c|}
\hline Gütergemeinschaft \\
\hline Gesamtgut von Ehefrau und Ehemann \\
\hline
\end{tabular}

\subsubsection{Gütertrennung}

Der ausserordentliche Güterstand der Gütertrennung ist für wirtschaftliche Schwierigkeiten vorgesehen (und tritt von Gesetzes wegen ein (Art. 188 ZGB) oder durch Anordnung des Richters (Art. 176, 185 und 189 ZGB) ${ }_{11}^{1}$ wird aber auch zur Haftungsabschottung von Unternehmern (und anderen Risikoträgern) verwendet. ${ }^{12}$ Dieser Güterstand kommt sodann für eingetragene Partner (registrierte, gleichgeschlechtliche Paare) grundsätzlich zur Anwendung. ${ }^{13}$

\begin{tabular}{|l|c|}
\hline \multicolumn{2}{|c|}{ Gütertrennung } \\
\hline Frauengut & Mannesgut \\
\hline
\end{tabular}

\subsubsection{Gestaltungsmöglichkeiten}

a. Die gesetzliche Regelung sieht beim Tod eines Ehegatten eine hälftige Teilung der Errungenschaft (Art. 215 Abs. 1 ZGB) bzw. des Gesamtguts (Art. 241 Abs. 1 ZGB) vor. Die Ehegatten können diese Zumeisung in einem Ehevertrag aber abweichend regeln (Art. 216 Abs. 1 ZGB bzw. Art. 241 Abs. 2 ZGB), so kann dem überlebenden Ehegatten im Sin-

10. Vgl. BK-Hausheer/Reusser/Geiser (Fn. 5), Vorbem. zu Art. 221 ff. ZGB N 39, welche daneben folgende weitere Charaktereigenschaften erwähnen: Gemeinsame Verwaltung und Kontrolle, gegenseitige Krediterhöhung und grössere Kooperationsbereitschaft (N 35-38)

11. CHK-Jungo (Fn. 1), Art. 181 ZGB N 4.

12. Vgl. Kurt Siehr, Wirtschaftliche Aspekte des Ehegüter- und Erbrechts aus Sicht der Europäischen Union, SZIER 13 (2004) 438.

13. Vgl. dazu Art. 18 ff. BG vom 18. Juni 2004 über die eingetragene Partnerschaft gleichgeschlechtlicher Paare (Partnerschaftsgesetz, PartG - SR 211.231); nach Art. 25 PartG besteht die Möglichkeit, sich in einem Vermögensvertrag der Errungenschaftsbeteiligung (vorne, 1.1.2) zu unterstellen. ne einer Meistbegünstigung das ganze Gesamtgut oder die ganze Errungenschaft zugewiesen werden. ${ }^{14}$ Diese Verfügung wird als Rechtsgeschäft unter Lebenden angesehen. ${ }^{15}$

b. Bei der Errungenschaftsbeteiligung wird die Zuwendung des (ganzen) Vorschlags durch Ehevertrag im Verhältnis zu den gemeinsamen Kindern toleriert, das heisst es kann vorkommen, dass nach der güterrechtlichen Auseinandersetzung gar kein Nachlass mehr vorhanden ist (weil kein Eigengut vorhanden ist) und pflichtteilsgeschützte Kinder somit in der Erbteilung leer ausgehen. Diese müssen bis zum Ableben des zweiten Ehegatten warten, bis sie ihr Erbe erhalten. ${ }^{16}$ Die Zuwendung des (ganzen) Vorschlags darf dagegen die Pflichtteile der nichtgemeinsamen Kinder nicht verletzen (Art. 216 Abs. 2 ZGB), zumal diese beim Ableben des zweitversterbenden Ehegatten (von Gesetzes wegen) leer ausgehen. Dies wird sichergestellt, indem die Pflichtteile der Kinder anhand der gesetzlichen (hälftigen) Teilung des Vorschlags ausgerechnet werden. ${ }^{17}$

c. Bei der Gütergemeinschaft besteht eine noch strengere Regelung, indem für die Berechnung der Pflichtteile gegenüber allen Nachkommen (nicht nur gegenüber den nichtgemeinsamen Kindern wie bei der Errungenschaftsbeteiligung $)^{18}$ von einer hälftigen Teilung des Gesamtguts ausgegangen wird (Art. 241 Abs. 3 $\mathrm{ZGB}) \cdot{ }^{19}$

\subsection{Gesetzliche Erbfolge}

a. Nach Abschluss der güterrechtlichen Auseinandersetzung steht fest, mie gross der Nachlass ist. Theoretisch kann es sein, dass gar kein Nachlass vorhanden ist, etwa wenn das ganze Gesamtgut in der Gütergemeinschaft dem überlebenden Ehegatten zugewiesen wird ${ }^{20}$ oder wenn der gesamte Vorschlag dem überlebenden Ehegatten überlassen wird und keine Eigengüter vorhanden sind. ${ }^{21}$

b. Eine empirische Untersuchung ${ }^{22}$ hat ergeben, dass im Jahre 200062.500 Erblasser Nachlässe im Umfang von etwa 28,5 Milliarden Franken hinterlassen

14. Vgl. dazu Hans Rainer Künzle, Einleitung, in: Praxiskommentar Erbrecht, hrsg. v. Daniel Abt und Thomas Weibel, 3. Aufl., Basel 2015 (zit. PraxKomm-Künzle), Einleitung, N 9; die Zuwendung kann in folgender Art erfolgen: (1) Festlegung konkreter Teilungsquoten, (2) Verzicht auf Vorschlagsbeteiligung, (3) Beschränkung der Vorschlagebeteiligung auf bestimmte Vermögenswerte und (4) Bestimmung konkreter Zuteilungssummen, vgl. dazu CHK-Jungo (Fn. 1), Art. 216-217 ZGB N 4.

15. Vgl. Philip R. Bornhauser, Die Rechtsnatur der Vorschlagszuweisung und deren Folge für die Erbteilung - Urteilanmerkung zu BGE 137 III 113, successio 5 (2011) 322.

16. Vgl. CHK-Jungo (Fn. 1), Art. 216-217 ZGB N 10, welche zu Recht bemerkt, dass die gemeinsamen Kinder beim Ableben des überlebenden Ehegatten 'mehr oder weniger' zum Zug kommen.

17. Die Pflichtteilberechnungsmasse besteht aus dem vorhandenen Nachlass und der hälftigen Errungenschaft beider Ehegatten, vgl. BSK ZGB-IHausheer/Aebi-Müller (Fn. 4), Art. 216 ZGB N 31.

18. Vgl. dazu vorne, 1.1.4. b)

19. Vgl. CHK-Jungo (Fn. 1), Art. 241 ZGB N 7.

20. Vgl. BGer. vom 19. Januar 2006 (5P.372/2005); vorne, 1.1.4

21. Vgl. vorne, 1.1.1.

22. Vgl. Heidi Stutz/Tobias Bauer/Susanne Schmugge, Erben in der Schweiz, Zürich/Chur 2007, S. 127 ff. 
haben, durchschnittlich also CHF 456.000 pro Erblasser. 159.500 Erben haben einen Betrag von durchschnittlich CHF 178.000 erhalten. Es stellt sich die Frage: Wie bedeutend sind Erbschaften volkswirtschaftlich? Es werden etwas weniger als 3\% des Volksvermögens jährlich vererbt. Die Erbschaften übersteigen das jährliche Sparen um etwa 30\%. Das zeigt, dass Erbschaften ein wichtiger wirtschaftlicher Faktor sind. Am häufigsten wird Bargeld vererbt $(66,1 \%)$, oft auch Liegenschaften $(28,0 \%)$, sodann Gebrauchsgegenstände und Erinnerungsstücke.

c. Der Ehegatte (bzw. eingetragene Partner) erbt neben Nachkommen die Hälfte, neben Eltern drei Viertel und neben weiter entfernten Verwandten den ganzen Nachlass (Art. 462 ZGB). Die Kinder erben zu gleichen Teilen und anstelle vorverstorbener Kinder treten deren Nachkommen in allen Graden nach Stämmen (Art. 457 ZGB). Die Stellung des überlebenden Ehegatten ist im Vergleich zu anderen Staaten ${ }^{23}$ angemessen; die entsprechenden Korrekturen wurden mit der 1988 in Kraft tretenden Revision vorgenommen. ${ }^{24} \mathrm{Zu}$ den Pflichtteilen siehe hinten, 2.

\subsection{Stellung in der Privat- und Sozialversicherung}

Bei kleineren Nachlässen spielen die verschiedenen Zweige (Säulen) der Privat- und Sozialversicherungen zunehmend eine wichtige Rolle, weil ein erheblicher Teil des vorhandenen Vermögens in diesen Gefässen (und nicht durch den Nachlass) auf die nächste Generation übertragen wird.

\subsubsection{Alters- und Hinterlassenenversicherung (erste Säule)}

Die Alters- und Hinterlassenenversicherung (AHV) ist eine staatliche Versicherung. Bei einer Scheidung erfolgt eine Halbierung der während der Ehe gesammelten Altersgutschriften unter den Ehegatten (Art. 29quinquies Abs. 4 lit. b Alters- und Hinterlassenenversicherung (AHVG)), ${ }^{25} \mathrm{im}$ Todesfall erhält der überlebende Ehegatte eine Witwen/Witwer-Rente (Art. 23 AHVG). ${ }^{26}$

\subsubsection{Berufliche Vorsorge (zmeite Säule)}

Die berufliche Vorsorge ist in der Schweiz obligatorisch. Bei einer Scheidung werden die Altersguthaben der beiden Ehegatten, die während der Ehe erarbeitet wurden, zwischen den Ehegatten geteilt (durch eine

23. Zu den Pflichtteilen anderer Länder (Belgien, Deutschland, Frankreich, Italien, Liechtenstein, Niederlande, Österreich, Schweden und Spanien, jeweils übersetzt in die deutsche Sprache und zwar in der Terminologie des ZGB) vgl. Hans Rainer Künzle/Albert Klöti/Christian Lyk/Daniel Schmid/Olivier Weber, Kendris Jahrbuch 2017/2018, Zürich 2017, S. $328 \mathrm{ff}$.

24. Vgl. AS 1986, 122.

25. Vgl. BG vom 20. Dezember 1946 über die Alters- und Hinterlassenenversicherung (AHVG - SR 831.10); das Jahr der Eheschliessung und der Auflösung der Ehe werden nicht berücksichtigt (Art. 50b Abs. $3 \mathrm{~V}$ vom 31. Oktober 1947 über die Alters- und Hinterlassenenversicherung (AHVV - SR 831.101)).

26. Thomas Ackermann, Fragen der ersten Säule und der Ergänzungsleistungen bei Scheidung und Trennung, AJP 25 (2016) 1459.
Ausgleichszahlung des einen Vorsorgewerkes an das andere - Art. 122 ZGB). ${ }^{27}$ Im Todesfall erhält der überlebende Ehegatte eine Witwenrente oder eine pauschale Abfindung (Art. 19 Abs. 1 und 2 Berufliche Vorsorge $(\mathrm{BVG}))^{28}$

\subsubsection{Lebensversicherung (dritte Säule)}

Bei allen Nachlässen gewinnen darüber hinaus Lebensversicherungen zunehmend an Bedeutung, weil dieses Instrument nicht mehr nur für die Risikoabdeckung (Art. 80 Versicherungsvertragsgesetz (VVG)), ${ }^{29}$ sondern auch für die Nachlassplanung verwendet wird. ${ }^{30}$ Der überlebende Ehegatte kann als Begünstigter einer Lebensversicherung eingesetzt werden, sowohl in der freien (Säule 3b) als auch in der gebundenen Lebensversicherung (Säule 3a). ${ }^{31}$

\subsubsection{Berücksichtigung in der Erbteilung}

Diese Leistungen der Privat- und Sozialversicherung ${ }^{32}$ gehen grundsätzlich am Nachlass vorbei, müssen aber in ausgewählten Fällen im Sinne von Ersatzforderungen mitberücksichtigt werden. Diese können zur Herabsetzung führen (Art. 527 ZGB). ${ }^{33} \mathrm{Zu}$ Einzelheiten siehe die nachfolgende Tabelle bzw. meine Ausführungen im Praxiskommentar Erbrecht. ${ }^{34}$

\section{Pflichtteile, Herabsetzung und Ausgleichung}

\subsection{Pflichtteile}

\subsubsection{Grundlagen}

a. Der Pflichtteil des Ehegatten und der Eltern beträgt je 1/2, derjenige der Kinder $3 / 4$ (Art. 471 ZGB). Während der Pflichtteil in den meisten Ländern als

27. Urs Gloor/Barbara Umbricht Lukas, Kommentar zu Art. 122-124 ZGB, in: Kommentar zum Schweizer Privatrecht, hrsg. v. Peter Breitschmid und Alexandra Jungo, 3. Aufl., Zürich/Basel/Genf 2016 (zit. CHKGloor/Umbricht Lukas), Art. 122 ZGB N 14.

28. Vgl. BG vom 25. Juni 1982 über die berufliche Alters-, Hinterlassenen und Invalidenvorsorge (BVG - SR 831.40); seit dem 1.1.2017 erhalten auch geschiedene Ehegatten unter bestimmten Voraussetzungen eine Leistung (Art. 19 Abs. 3 BVG); Esther Amstutz, Die Begünstigungsordnung der beruflichen Vorsorge, Zürich 2014, S. 28 ff. und $38 \mathrm{f}$.

29. Im Falle eines überschuldeten Nachlasses, welcher ausgeschlagen wird (Art. 566 ff. ZGB), kann der überlebende Ehegatte seinen Anspruch auf die Versicherungsleistung direkt gegenüber dem Versicherer geltend machen und dieser Anspruch kann nicht gepfändet werden, was eine wirksame Asset Protection ist.

30. Vgl. Hans Rainer Künzle, Lebensversicherung als Instrument der Nachlassplanung, in: Festschrift für Moritz Kuhn, hrsg. v. Rolf Dörig etc., Zürich 2009, S. $245 \mathrm{ff}$.

31. Vgl. Regina Elisabeth Aebi-Müller, Säule $3 a$ im Güter- und Erbrecht, in: Familienvermögensrecht - Symposium zum Familienrecht, Zürich 2016, S. 65 f.; die beiden Säulen unterscheiden sich durch die steuerliche Behandlung, ehegüterrechtlich und erbrechtlich sind sie gleichgestellt.

32. Vgl. vorne, 1.3.1-1.3.3.

33. Vgl. dazu hinten, 2.2.

34. Vgl. PraxKomm-Künzle (Fn. 14), Einleitung N $102 \mathrm{ff}$ 


\begin{tabular}{|c|c|c|c|}
\hline $\begin{array}{l}\text { Leistungen an Erben/Zurech- } \\
\text { nung beim Erblasser }\end{array}$ & $\begin{array}{l}\text { Güterrechtliche Auseinander- } \\
\text { setzung (Art. } 204 \text { ff. ZGB) }\end{array}$ & $\begin{array}{l}\text { Bestandteil des Nachlasses } \\
\text { (Art. } 560 \text { ZGB) }\end{array}$ & Herabsetzung (Art. 527 ZGB) \\
\hline $\begin{array}{l}\text { Säule 1: AHV (Witen und Wai- } \\
\text { sen) /EO (keine Leistung) }\end{array}$ & Nein & Nein & Nein \\
\hline $\begin{array}{l}\text { Säule 1: ELG (Ergänzungsleis- } \\
\text { tungen an den Erblasser) }\end{array}$ & - & $\begin{array}{l}\text { Rückforderung zu Unrecht } \\
\text { bezo- gener Leistungen }\end{array}$ & - \\
\hline $\begin{array}{l}\text { Säule 1: (Sozialhilfe an den } \\
\text { Erblasser) }\end{array}$ & - & $\begin{array}{l}\text { Rückforderung rechtmässig } \\
\text { bezo- gener Leistungen }\end{array}$ & - \\
\hline Säule 2: Patronale Stiftung & Nein & Nein & Nein \\
\hline $\begin{array}{l}\text { Säule 2a: Obligatorium (Pensi- } \\
\text { onskasse/ Sammelstiftung) }\end{array}$ & Nein & Nein & Nein \\
\hline $\begin{array}{l}\text { Säule 2a: Obligatorium (Freizü- } \\
\text { gigkeitskonto oder -police) }\end{array}$ & Nein & Nein & Nein \\
\hline $\begin{array}{l}\text { Säule 2b: Überobligatorium } \\
\text { (Pensionskasse/ Sammelstif- } \\
\text { tung) }\end{array}$ & Nein & $\begin{array}{l}\text { Nein (ausnahms- weise ja, } \\
\text { wenn individuell vereinbart) }\end{array}$ & $\begin{array}{l}\text { Nein (ausnahmsweise ja, wenn } \\
\text { individuell vereinbart) }\end{array}$ \\
\hline $\begin{array}{l}\text { Säule 2b: Überobligatorium } \\
\text { (Freizügigkeitskonto oder } \\
\text {-police) }\end{array}$ & Nein & Nein & Nein \\
\hline $\begin{array}{l}\text { Säule 3a: Gebundene Lebens- } \\
\text { versicherung }\end{array}$ & Ja (Rückkaufswert) & Nein & Ja (Rückkaufswert) \\
\hline $\begin{array}{l}\text { Säule 3a: Gebundenes Bank- } \\
\text { sparen }\end{array}$ & Ja (Austrittsleistung) & Nein & Ja (Austrittsleistung) \\
\hline $\begin{array}{l}\text { Säule 3b: Freie Lebensversiche- } \\
\text { rung }\end{array}$ & Ja (Rückkaufswert) & $\begin{array}{l}\text { Nein (wenn direkter Forde- } \\
\text { rungsanspruch eines Begünstig- } \\
\text { ten) }\end{array}$ & Ja (Rückkaufswert) \\
\hline Säule 3b: Freies Banksparen & $\mathrm{Ja}$ & Ja & Ja \\
\hline
\end{tabular}

Bruchteil der gesamten Erbmasse ausgedrückt wird, ${ }^{35}$ bezieht er sich in der Schweiz auf den gesetzlichen Erbteil. ${ }^{36}$ Wer auf den Pflichtteil gesetzt wird, bleibt Erbe (Mitglied der Erbengemeinschaft) ${ }^{37}$ und erscheint somit auch auf dem Erbschein (von Art. 559 ZGB als 'Erbbescheinigung' bezeichnet). Der Pflichtteil muss allerdings nur 'dem Werte nach'

Das trifft zum Beispiel zu auf Belgien, Frankreich, Italien, Niederlande und Spanien, vgl. Künzle/Klöti/Lyk/Schmid/Weber (Fn. 23), S. $350 \mathrm{ff.}$ $399 \mathrm{ff}$., $418 \mathrm{ff}$., $488 \mathrm{ff}$. und $558 \mathrm{ff}$.

36. Zum gesetzlichen Erbteil vgl. vorne, 1.2

37. Vgl. Christoph Nertz, Kommentar zu Art. 470-476 ZGB, in: Praxiskommentar Erbrecht, hrsg. v. Daniel Abt und Thomas Weibel, 3. Aufl., Basel 2015 (zit. PraxKomm-Nertz) Art. 470 ZGB N 10; anders etwa die Regelung des deutschen Rechts, wo der Pflichtteilsberechtigte nicht mehr Mitglied der Erbengemeinschaft ist, nur noch einen obligatorischen Anspruch gegenüber den Erben hat, sein Auskunftsrecht nach § 2314 BGB gegenüber den Erben geltend machen muss (welche womöglich Zuwendungen vom Erblasser erhalten haben) und sich also nicht direkt an Banken wenden kann, weil er nicht auf dem Erbschein steht, vgl. dazu etwa Christopher Riedel, Kommentar zu §§ 2303-2314 BGB, in: Praxiskommentar Erbrecht, hrsg. v. Jürgen Damrau, 3. Aufl., Bonn 2014, § 2314 BGB N 2 ff. abgefunden werden und es besteht kein Naturalanspruch (Art. 522 Abs. 1 ZGB). ${ }^{38}$

b. Der Pflichtteil des Ehegatten (1/2) scheint angemessen ${ }^{39}$ während derjenige der Eltern $(1 / 2)$ heute kaum mehr seine Berechtigung hat, weil die meisten Eltern auf dieses Geld nicht angewiesen sind bzw. vor den Kindern sterben. ${ }^{40}$ Der Pflichtteil der Kinder erscheint im internationalen Vergleich als hoch, üblich ist $1 / 2$ und nicht $3 / 4{ }^{41}$

c. Gegenwärtig ist eine Revision des Erbrechts im Gange, bei welcher vorgeschlagen wurde, den Pflichtteil der Kinder von $3 / 4$ auf $1 / 2$ zu reduzieren sowie den

38. Vgl. Stephanie Hrubesch-Millauer, Kommentar zu Art. 522-533 ZGB, in: Praxiskommentar Erbrecht, hrsg. v. Daniel Abt und Thomas Weibel, 3. Aufl., Basel 2015 (zit. PraxKomm-Hrubesch-Millauer), Art. 522 ZGB N 2

39. Siehe aber die Motion 06.3656 (Nationalrätin Laura Sadis): Erbberechtigung des überlebenden Ehegatten, vgl. www.parlament.ch/d/cvgeschaefte?gesch_id=20063656.

40. Vgl. Urs Schenker, Strukturierung der Unternehmensnachfolge bei der Aktiengesellschaft, in: Entwicklungen im Gesellschaftsrecht XII, Zürich 2017, S. 229 Fn. 30: 'In der Praxis hat der Pflichtteil der Eltern allerdings kaum je eine Bedeutung, da diese nur selten den Erblasser überleben'

41. Zu den Gesetzestexten des Erbrechts anderer Länder vgl. die Rubrik 'Gesetze', 'Ausland' bei www.successio.ch. 


\begin{tabular}{|c|c|c|c|c|c|}
\hline Erben (Konstellation) & $\begin{array}{l}\text { Gesetzlicher Erb- } \\
\text { teil (A) }\end{array}$ & Pflichtteil (B) & $\begin{array}{l}\text { Geschützter Erb- } \\
\text { teil }(A \times B) \text { ein- } \\
\text { zeln }\end{array}$ & $\begin{array}{l}\text { Geschützter Erb- } \\
\text { teil }(A \times B) \text { total }\end{array}$ & Verfügbare Quote \\
\hline $\begin{array}{l}\text { Ehegatte (bzw. eingetragener } \\
\text { Partner) allein }\end{array}$ & $1 / 1$ & $1 / 2$ & $1 / 2$ & $1 / 2$ & $1 / 2$ \\
\hline Kinder (allein) & $1 / 1$ & $3 / 4$ & $3 / 4$ & $3 / 4$ & $1 / 4$ \\
\hline $\begin{array}{l}\text { Beide Eltern/ein Elternteil } \\
\text { (allein) }\end{array}$ & $1 / 1$ & $1 / 2$ & $1 / 2$ & $1 / 2$ & $1 / 2$ \\
\hline Ehegatte & $1 / 2$ & $1 / 2$ & $1 / 4$ & & \\
\hline+ & & & & $5 / 8$ & $3 / 8$ \\
\hline $\begin{array}{l}\text { ein oder mehrere Nachkom- } \\
\text { men }\end{array}$ & $1 / 2$ & $3 / 4$ & $3 / 8$ & & \\
\hline Ehegatte & $3 / 4$ & $1 / 2$ & $3 / 8$ & & \\
\hline+ & & & & $1 / 2$ & $1 / 2$ \\
\hline Beide Eltern/ein Elternteil & $1 / 4$ & $1 / 2$ & $1 / 8$ & & \\
\hline Ehegatte & $3 / 4$ & $1 / 2$ & $3 / 8$ & & \\
\hline \multicolumn{6}{|l|}{+} \\
\hline Ein Elternteil & $1 / 8$ & $1 / 2$ & $1 / 16$ & $7 / 16$ & $9 / 16$ \\
\hline \multicolumn{6}{|l|}{+} \\
\hline Bruder/Schwester & $1 / 8$ & - & & & \\
\hline Ehegatte & $3 / 4$ & $1 / 2$ & $3 / 8$ & & \\
\hline+ & & & & $3 / 8$ & $5 / 8$ \\
\hline Bruder/Schwester & $1 / 4$ & - & - & & \\
\hline
\end{tabular}

Pflichtteil der Eltern von gegenwärtig 1/2 aufzuheben. ${ }^{42}$ Da diese Vorschläge in der Vernehmlassung nur teilweise auf Zustimmung gestossen sind, ${ }^{43}$ ist gegenwärtig unklar, ob diese Vorschläge auch umgesetzt werden.

d. Pflichtteile stehen bei Erbschaftsplanungen häufig im Wege. Dieses Hindernis kann dadurch beseitigt werden, dass ein Erbe auf seinen Pflichtteil in einem Erbvertrag verzichtet (Art. 495 ZGB). ${ }^{44}$ Üblicherweise wird er für diesen Verzicht (gleichzeitig) eine Zuwendung erhalten. Da sich der Verzicht auf einen erst in der Zukunft bestimmbaren Anspruch bezieht, ${ }^{45}$ wird weder eine umfassende Offenlegung der Vermögensverhältnisse des Erblassers verlangt, noch besteht unter solchen Umständen Aussicht darauf, dass spä-

42. Vgl. Zum Entwurf vgl. www.bj.admin.ch/dam/data/bj/gesellschaft/ gesetzgebung/erbrecht/entw-d.pdf; zum Botschaft vgl. www.bj.admin. ch/dam/data/bj/gesellschaft/gesetzgebung/erbrecht/bot-d.pdf.

43. Zu den Vernehmlassungsergebnissen vgl. www.bj.admin.ch/dam/data/ bj/gesellschaft/gesetzgebung/erbrecht/ve-ber-d.pdf. Das Parlament wird sich demnächst mit der Vorlage befassen und frühestens 2020 ist mit einer Inkraftsetzung zu rechnen.

44. Vgl. Stephanie Hrubesch-Millauer, Der Erbvertrag: Bindung und Sicherung des (letzten) Willens des Erblassers, Zürich/St. Gallen 2008, S. 39 f.

45. Zum Todestagsprinzip, das auch bei der Ausgleichung zur Anwendung kommt, vgl. hinten, 2.3. d). ter erfolgreich Irrtum oder veränderte Umstände (clausula rebus sic stantibus) geltend gemacht werden können, weil dies bei blossen Wünschen und Hoffnungen nicht zutrifft. ${ }^{46}$

\subsubsection{Beispiel einer Erbschaftsplanung}

a. Sachverhalt: Claude (55), und Sandra, (47), führen ein Unternehmen. Sie haben zwei Kinder, Philip (24) und Patricia (23). Das Vermögen beträgt heute CHF 1.500.000. Als Startkapital für das Unternehmen wurden Beträge von CHF 250.000 (Ehemann) und CHF 100.000 (Ehefrau) verwendet, welche aus jeweiligem Eigengut (EGM, EGF) stammen (vor der Ehe gespartes Vermögen bzw. Zuwendungen der Eltern).

46. Ähnlich OLG Düsseldorf I-3 Wx 193/12 vom 21.02.2013, ZErb 15 (2013) 94 (Ehegatte unterzeichnet Erbverzicht, ohne nach den Vermögensverhältnissen zu fragen: Das Verschweigen eines Auslandkontos über EUR 300,000 macht den Erbvertrag unter diesen Umständen nicht sittenwidrig); in der Schweiz gibt es keine höchstgerichtliche Rechtsprechung; nach Stefan Grundmann, Kommentar zu Art. 494-497 ZGB, in: Praxiskommentar Erbrecht, hrsg. v. Daniel Abt und Thomas Weibel, 3. Aufl., Basel 2015, Art. 495 ZGB N 4 ist 'in Extremfällen' die Anrufung der clausula rebus sic stantibus denkbar; Hrubesch-Millauer (Fn. 44), S. 329, weist darauf hin, dass dieses Thema in der Rechtsprechung und Lehre nur wenig behandelt wird. 


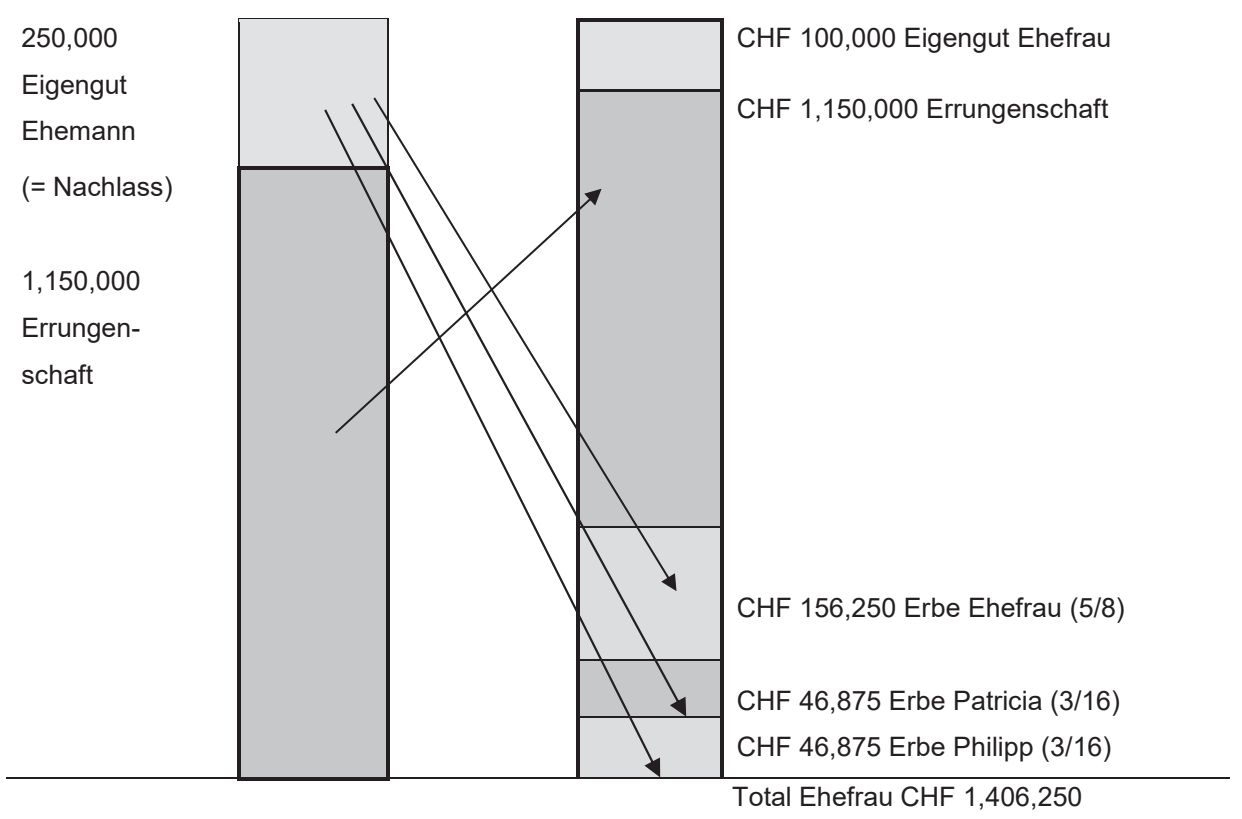

b. Erbschaftsplanung: Es ist vorgesehen, dass die Ehefrau den Betrieb weiterführt, falls der Ehemann versterben sollte. Deshalb schliessen die Ehegatten einen Ehevertrag $\mathrm{ab}$, in welchem sie den gesamten Vorschlag dem überlebenden Ehegatten zuweisen. In einem Testament setzen zudem beide Ehegatten ihre Kinder jeweils auf den Pflichtteil.

c. Dieses Beispiel zeigt, dass die verfügbare Masse - mindestens bei der vorliegenden Konstellation, die typisch ist - im Eherecht pesentlich grösser ist (CHF 575.000) als im Erbrecht (CHF 31.250) und dass man dem Ehevertrag deshalb vermehrt Aufmerksamkeit schenken sollte. Das Beispiel zeigt aber auch, dass bei einer Unternehmensnachfolge durch einen Ehegatten nicht immer der ganze Spielraum ausgenützt werden muss, um das Unternehmen weiterführen zu können. Wenn eines von mehreren Kindern das Unternehmen weiterführen soll, erweisen sich die Pflichtteile dagegen regelmässig als grosses Hindernis. Dann ist mit verschiedenen Instrumenten (wie Mehr- und Minderheitsanteilen mit Aktienbindungsvertrag, Unternehmensbewertung, Pflichtteilsverzicht) zu arbeiten.

\subsection{Herabsetzung}

a. Die Herabsetzung bezweckt den Schutz der Pflichttei$l e^{47}$ und damit den Schutz der gesetzlichen Erbfolge. ${ }^{48}$ Sowohl der vorhandene Nachlass als auch die unentgeltlichen Zuwendungen zu Lebzeiten, welche $\mathrm{zu}$ diesem hinzugerechnet werden, sind zum Verkehrswert zu bewerten. ${ }^{49}$

47. Vgl. dazu vorne, 2.1 .

48. Vgl. dazu vorne, 1.2.

49. Vgl. Paul Eitel/Marjolein Bieri, Die Durchführung der Herabsetzung bei Schenkungen, Lebensversicherungen und Trusts, successio 9 (2015) 289. b. Zuwendungen unter Lebenden (in erster Linie Schenkungen, aber auch Leistungen aus Lebensversicherungen), ${ }^{50}$ welche in den letzten 5 Jahren vor dem Tod des Erblassers erfolgt sind, unterstehen der Herabsetzung (Art. 527 Ziff. 3 ZGB).$^{51}$ Bei Schenkungen wird zum Schutz der Pflichtteilsberechtigten der volle Betrag (bei der Lebensversicherung der Rückkaufswert - Art. 529 ZGB) ${ }^{52}$ hinzugerechnet (Herabsetzungs-Berechnungsmasse). ${ }^{53}$

c. Ohne zeitliche Grenze erfolgt die Herabsetzung von lebzeitigen Zuwendungen, wenn dem Erblasser Umgehungsabsichten nachgewiesen werden können (Art. 527 Ziff. 4 ZGB). ${ }^{54}$ Keine Herabsetzung erfolgt, wenn die Zuwendung der Ausgleichung (nachfolgend B III) unterliegt. ${ }^{55}$

d. Beispiel: Das Kind A bekam zu Lebzeiten eine Wohnung zum damaligen Wert von CHF 700.000. Diese

50. Nach herrschender Lehre (vgl. etwa PraxKomm-Nertz (Fn. 37), Art. 476 ZGB N 24) und Rechtsprechung (BGE 112 || 157) sind Begünstigungen in Lebensversicherungen als Verfügungen unter Lebenden anzusehen.

51. Bei der Berechnung der 5-Jahres-Frist wird auf den Zeitpunkt des Vollzugs der Schenkung abgestellt und nicht etwa auf den Zeitpunkt des Schenkungsversprechens, vgl. Eitel/Bieri (Fn. 49), successio 9 (2015) 293 ff.; bei einem Trust ist auf den Zeitpunkt der Zuwendung an den Trust abzustellen (301).

52. Die Hinzurechnung erfolgt unter der Voraussetzung, dass der Begünstigte die Versicherungsleistung unentgeltlich erworben hat (durch Begünstigung, Abtretung oder letztwillige Verfügung), vgl. PraxKommNertz (Fn. 37), Art. 476 ZGB N 24 ff.; Künzle (Fn. 30), S. 247 f.

53. Vgl. BGE 102 II 325 f.; Alfred Reber/Thomas Meili, Todesfallleistungen aus über- und ausserobligatorischer beruflicher Vorsorge und Pflichtteilsschutz, SJZ 92 (1996) 121.

54. Das Bundesgericht hat eine solche Umgehungsabsicht in BGE 128 III 314 E. 4 in der ehevertraglichen Vorschlagszuweisung in einem Ehevertrag (vgl. dazu vorne, 1.1.4) gesehen.

55. Vgl. Jacqueline Burckhardt Bertossa, Kommentar zu 626-632 ZGB, in: Basler Praxiskommentar Erbrecht, hrsg. v. Daniel Abt und Thomas Weibel, 3. Aufl., Basel 2015 (zit. PraxKomm-Burckhardt Bertossa), Vorbem. zu Art. $626 \mathrm{ff}$. ZGB N 9 f. 
Wohnung ist heute CHF 1.100 .000 wert. Der vorhandene Nachlass beträgt CHF 500.000. Es sind zwei Kinder vorhanden, A und B. B wurde auf den Pflichtteil gesetzt (3/8). Die Pflichtteilberechnungsmasse beträgt $\mathrm{CHF} 1.600 .000$ (Verkehrswert per Todestag), der Pflichtteil von B (3/8) somit CHF 600.000. Das vorhandene Vermögen reicht nicht aus, um ihm den Pflichtteil zu geben, somit hat A zusätzlich CHF 100.000 an B zu bezahlen.

e. Die Herabsetzung weist verschiedene Stolpersteine auf: Wie das vorne dargestellte Beispiel ${ }^{56}$ zeigt, können Wertsteigerungen von Liegenschaften zu einem Stolperstein in der Erbschaftsplanung werden, wenn sie unbeabsichtigt zu Herabsetzungsansprüchen führen. ${ }^{57}$ Die Empfänger von lebzeitigen Zuwendungen sind sich dieser Problematik regelmässig nicht bewusst und sie haben auch keine Informationen über die Reihenfolge der herabsetzbaren Zuwendungen. Wenn ein Erblasser zum Beispiel eine gemeinnützige Stiftung errichten und Zuwendungen an sie vornehmen will, sollte er einen Erbverzicht von allen pflichtteilsgeschützten Erben einholen. ${ }^{58}$

\subsection{Ausgleichung}

a. Die Ausgleichung bezweckt die Verwirklichung einer Gleichheits- oder Gerechtigkeitsidee. ${ }^{59}$ Damit wird eine Gleichbehandlung der Nachkommen im Rahmen der gesetzlichen Erbteilung angestrebt, welche allerdings durch eine anders lautende Anordnung des Erblassers durchbrochen werden kann (Art. 626 Abs. 2 ZGB). ${ }^{60}$ Eine Befreiung von der Ausgleichung erfolgt entweder im Testament des Erblassers oder dann im Vertrag, mit welchem die (unentgeltliche) Zuwendung geregelt wird (häufig ein Schenkungsvertrag). In letzterem Fall ist sie formlos gültig. ${ }^{61}$

b. Eine Ausgleichung nach Artikel 626 Absatz 1 ZGB erfolgt durch die gesetzlichen Erben, eine solche nach Artikel 626 Absatz 2 ZGB dagegen (nur) durch die Nachkommen. Der überlebende Ehegatte ist nach Artikel 626 Absatz 1 ZGB ausgleichspflichtig, wenn der Erblasser dies angeordnet hat. Bei der gesetzlichen Ausgleichung nach Artikel 626 Absatz 2 ZGB ist der überlebende Ehegatte nach der Rechtsprechung des Bundesgerichts $^{62}$ Gläubiger, aber er kann nicht Schuldner dieser Ausgleichung sein, was von der herrschenden Lehre kritisiert wird. ${ }^{63}$

56. Vgl. vorne, 2.2. d)

57. Vgl. René Strazzer, Ein erbrechtlicher Stolperstein, NZZ vom 7. Mai 2018, S. 12.

58. In gewissen Ländern sind gemeinnützige Zuwendungen von der Herabsetzung befreit.

59. Vgl. Paul Eitel, Berner Kommentar, Band III: Das Erbrecht, 2. Abteilung: Der Erbgang, 3. Teilband: Die Ausgleichung (Art. 626-632 ZGB), Bern 2004 (zit. BK-Eitel), Vorbem. vor Art. 626 ff. ZGB N 10.

60. Vgl. Peter Breitschmid/Paul Eitel/Roland Fankhauser/Thomas Geiser/ Alexandra Jungo, Erbrecht (litera B), 3. Aufl., Zürich 2016, S. 158.

61. Vgl. PraxKomm-Burckhardt Bertossa (Fn. 55), Art. 626 ZGB N 69, mit Verweis auf BGE 69 II 71 E. 2).

62. Vgl. BGE 51 II 378 E. 1; BGE 77 II 228 E. 3c; BGer. 5A_141/2007 vom 21.12.2007 E. 9.2.

63. Vgl. PraxKomm-Burckhardt Bertossa (Fn. 55), Art. 626 ZGB N 17 ff., welche darauf hinweist, dass die Gläubigerstellung des überlebenden c. In Artikel 626 Absatz 1 ZGB werden lebzeitige Zumendungen ausgeglichen, in Artikel 626 Absatz 2 ZGB dagegen (vor allem) Ausstattungen und Vermögenszuwendungen. Es handelt sich dabei um Zuwendungen von einer gewissen Bedeutung (Grosszumendungen $).{ }^{64}$

d. Die Ausgleichung erfolgt zum Wert der Zuwendung im Zeitpunkt des Erbgangs (Todestagsprinzip, Art. 630 ZGB). Dies bedeutet, dass eine Geldzuwendung beim Ableben des Erblassers den gleichen Wert hat wie bei der Zuwendung (Nominalwertprinzip), ${ }^{65}$ während bei Liegenschaften regelmässig ein Wertzuwachs zu verzeichnen ist. ${ }^{66}$

e. Der Ausgleichungsschuldner kann wählen, ob die Ausgleichung dem Werte nach oder in natura (durch Wiedereinbringung des zugewendeten Gegenstands) erfolgen soll (Art. 628 ZGB). ${ }^{67}$ Er kann sich der Ausgleichung entziehen, wenn er sein Erbe ausschlägt (Art. 566 ZGB). ${ }^{68}$ Dies kann allerdings (wegen seines Wegfalls als Erbe) zu massiv höheren Herabsetzungsansprüchen der verbliebenen Erben führen, weil sich deren Pflichtteil vergrössert hat, wie das nachfolgende Beispiel zeigt.

f. Beispiel: Der Erblasser hinterlässt zwei Kinder (A und B). Bei seinem Ableben ist er vermögenslos. Er hat aber A zu Lebzeiten CHF 600.000 geschenkt, welche auszugleichen wären. Wenn A das Erbe annimmt, muss $\mathrm{A}$ an $\mathrm{B} \mathrm{CHF} 300.000$ herausgeben. In der Meinung, dies vermeiden zu können, schlägt A die Erbschaft aus. Nun hat $B$ als alleiniger Erbe einen Pflichtteil von 3/4 (statt wie bisher 3/8) und kann somit eine Herabsetzung im Umfang von CHF 450.000 verlangen.

g. Das Ausgleichsrecht weist verschiedene Stolpersteine auf: Ein und derselbe Gegenstand kann bei Anwendung der bundesgerichtlichen Versorgungskollation (Ausstattung und so weiter) je nach der Situation des Empfängers unter Artikel 626 Absatz 1 oder Absatz 2 ZGB fallen und im einen Fall ausgleichspflichtig sein und im anderen nicht. ${ }^{69}$ Problematisch ist sodann, wenn einem Kind ein Grundstück unentgeltlich übertragen wird (und es den Mehrwert teilen muss) und dem anderen (im gleichen Umfang) Geld, um ebenfalls ein Grundstück zu kaufen (Nominalwertprinzip).$^{70}$ Die Nutzung einer Liegenschaft muss im Falle einer unentgeltlichen Überlassung ausgeglichen werden, im Falle einer unentgeltlichen Zuwendung

Ehegatten besonders dann stossend ist, wenn sie in zweiter Ehe verheiratet war und Ausgleichung von Zuwendungen verlangt, welche an die Kinder aus erster Ehe vor ihrer Heirat vorgenommen wurden (N 22).

64. Vgl. Breitschmid/Eitel/Fankhauser/Geiser/Jungo (Fn. 60), S. 162.

65. Vgl. PraxKomm-Burckhardt Bertossa (Fn. 55), Art. 630 ZGB N 9.

66. Massgebend ist ein Zuwachs des konjunkturellen Verkehrswerts, während industrielle (selbst erarbeitete) Wertveränderungen nicht zu berücksichtigen sind, vgl. BGE 133 III 416 E. 6.3.4; BK-Eitel (Fn. 59), Art. 630 ZGB N 6.

67. Vgl. BK-Eitel (Fn. 59), Art. 628 ZGB N 13 ff. (Wahlrecht).

68. Vgl. BK-Eitel (Fn. 59), Art. 626 ZGB N 9; PraxKomm-Burckhardt Bertossa (Fn. 55), Art. 626 ZGB N 7.

69. Vgl. PraxKomm-Burckhardt Bertossa (Fn. 55), Art. 626 ZGB N 51 und 105.

70. Vgl. dazu vorne, 2.3. d). 
dagegen nicht. ${ }^{71}$ Es empfiehlt sich in jedem Fall, dass der Erblasser die Frage der Ausgleichspflicht (im Schenkungsvertrag, Testament oder Erbvertrag) regelt.

\section{Internationales Erbrecht}

Das schweizerische internationale Erbrecht ist in Artikel 86-96 Bundesgesetz über das Internationale Privatrecht $(\text { IPRG })^{72}$ geregelt. Diese Regelungen werden gegenwärtig revidiert und an die Europäische Erbrechtsverordnung (EuErbVO ${ }^{73}$ angepasst. Die Motion 14.4285 Luc Recordon, welche dies verlangte, wurde vom Nationalrat zwar am 21.9.2015 abgelehnt, aber das Bundesamt für Justiz hat 2016 selbständig eine Revision von Art. 86-96 IPRG in Angriff genommen, um (neben einer Harmonisierung mit der EuErbVO) die Gesetzgebung auf den neusten Stand zu bringen. Zunächst wurden 21 mögliche Änderungen in Betracht gezogen, von denen nachfolgend einige behandelt werden. Danach hat eine Expertenkommission ${ }^{74}$ einen Vorentwurf ${ }^{75}$ und Bericht ${ }^{76}$ erarbeitet, welche vom Bundesrat am 12. Februar 2018 publiziert wurden. Nach der Vernehmlassung, welche bis zum 31. Mai 2018 lief, wird nun vom Bundesamt für Justiz (unter Einbindung der Expertenkommission) ein Entwurf ausgearbeitet, welchen das Parlament (National- und Ständerat) etwa 2019 behandeln wird. Die Neuregelung dürfte etwa 2020/2021 in Kraft treten. Da in den Niederlanden die EuErbVO gilt, ist eine solche Anpassung für Personen, welche einen Bezug zu beiden Ländern haben, von grossem Interesse.

\subsection{Hauptanknüpfung: Wohnsitz}

a. Sowohl die Zuständigkeit (Art. 86 IPRG) ${ }^{77}$ als auch das anwendbare Recht (Art. 90 IPRG) knüpfen grundsätzlich am letzten Wohnsitz des Erblassers an. ${ }^{78}$ Dies entspricht weitgehend der EU-Erbrechtsverordnung, wo an den letzten gewöhnlichen Aufenthalt des Erblassers angeknüpft wird (Art. 4 und 21 EuErbVO).

b. Es wurde überlegt, ob der letzte Wohnsitz in verschiedenen Bestimmungen ${ }^{79}$ durch den letzten gewöhn-

71. Vgl. PraxKomm-Burckhardt Bertossa (Fn. 55), Art. 626 ZGB N 108 und Art. 629 ZGB N 34

72. Vgl. BG vom 18. Dezember 1987 über das Internationale Privatrecht (IPRG, SR 291)

73. Vgl. Verordnung (EU) Nr. 650/2012 des Europäischen Parlaments und des Rates vom 4. Juli 2012 über die Zuständigkeit, das anwendbare Recht, die Anerkennung und Vollstreckung von Entscheidungen und die Annahme und Vollstreckung öffentlicher Urkunden in Erbsachen sowie zur Einführung eines Europäischen Nachlasszeugnisses (EuErbVO).

74. Der Expertenkommission gehörten neben mir auch Prof. Andrea Bonomi, Prof. Barbara Graham-Siegenthaler und Dr. Kinga M. Weiss an.

75. Vgl. www.ejpd.admin.ch/dam/data/bj/aktuell/news/2018/2018-02-14 /vorentw-d.pdf.

76. Vgl. www.ejpd.admin.ch/dam/data/bj/aktuell/news/2018/2018-02-14 /vn-ber-d.pdf.

77. Vgl. Hans Rainer Künzle, Kommentar zu Art. 86-96 IPRG, in: Zürcher Kommentar, Zürich 2018 (zit. ZK-Künzle), Art. 86 IPRG N 2 ff.

78. Vgl. ZK-Künzle (Fn. 77), Art. 90 IPRG N 2 ff.

79. Es geht um Art. 86-91, 94, 95 IPRG. lichen Aufenthalt ersetzt werden sollte. Dies erschien aus mehreren Gründen nicht ratsam. Zum einen ist noch nicht ganz geklärt, was der gewöhnliche Aufenthalt wirklich ist. ${ }^{80}$ Noch wichtiger ist aber, dass der Wohnsitz nicht nur in Artikel 86-96 IPRG, sondern auch an vielen anderen Orten (insbesondere beim ehelichen Güterrecht, Art. 51 ff. IPRG) verwendet wird und man ein gut funktionierendes System nicht auseinanderreissen sollte. Zudem weist der Wohnsitz (Art. 20 IPRG) eine subjektive Komponente auf (Absicht des dauernden Verbleibs), welche dem Erblasser eine gewisse Sicherheit in der Erbschaftsplanung verschafft, nämlich wenn er im Testament feststellt, dass er an einem bestimmten Ort seinen Wohnsitz hat und daran die Zuständigkeit und/oder das anwendbare Recht anknüpfen möchte. $^{81}$

c. In Artikel 86 Absatz 2 IPRG wird die ausschliessliche Zuständigkeit für ausländische Grundstücke vorbehalten. ${ }^{82}$ Die Zuständigkeit im Ausland führt regelmässig auch zur Anwendung des ausländischen Erbrechts. ${ }^{83}$ Nach herrschender Meinung ist eine durch Artikel 86 Absatz 2 IPRG verursachte, vom schweizerischen Erbrecht abweichende, Erbteilung bei ausländischen Grundstücken im Inland rechnerisch anzurechnen und zwar sowohl im Falle der Nachlassspaltung als auch im Falle des Nachlasskonflikts. ${ }^{84}$ Abgelehnt wird dagegen eine Ausgleichung von ausländischen Grundstückszuteilungen, welche dazu führen würde, dass im Inland etwas in den Nachlass einzuschiessen wäre. ${ }^{85}$

80. Das zeigt etwa die deutsche Rechtsprechung zu diesem Thema, vgl. KG 1 AR 8/16 vom 26.04.2016, IPRax 38 (2018) 29 und ZErb 18 (2016) 199 (Wohnung einer Berliners in einer Lagerhalle in Polen); OLG Hamburg 2 W 85/16 vom 16.11.2016, www.landesrechthamburg.de/jportal/portal/page/bsharprod.psml?showdoccase=1\&doc. id=KORE502652017\&st=ent (getrennt in Spanien lebender Hamburger); OLG München 31 AR 47/17 vom 22.03.2017, ErbR 12 (2017) 356 und ZEV 2017, 333 (letzter Aufenthalt in einem Pflegeheim, ohne Abklärung ob ein Bleibewille bestand bzw. noch möglich war).

81. Vgl. ZK-Künzle (Fn. 77), Vorbem. zu Art. 86-96 IPRG N 89; wie der in Fn. 80 erwähnte Entscheid des OLG München 31 AR 47/17 vom 22.03.2017 zeigt, ist der letzte gewöhnliche Aufenthalt nicht rein objektiv bestimmbar (die Geschäftsfähigkeit spielt bei der Bildung eines Bleibewillens eine Rolle), aber das subjektive Element ist von untergeordneter Bedeutung.

82. Dies ist der Fall in Staaten wie Albanien, Argentinien, Australien, Bolivien, China (Volksrepublik), Ghana, Indien, Irland, Kanada, Kasachstan, Malaysia, Monaco, Neuseeland, Nigeria, Pakistan, Paraguay, Russland, San Marino, Südafrika, Thailand, Türkei, Ukraine, Usbekistan, Vereinigte Staaten von Amerika, Vereinigtes Königreich und Weissrussland (Belarus), vgl. ZK-Künzle (Fn. 77), Art. 86 IPRG N 20.

83. Vgl. ZK-Künzle (Fn. 77), Art. 90 IPRG N 5.

84. Vgl. Anton K. Schnyder/Manuel Liatowitsch, Kommentar zu Art. 86-96 IPRG, in: Basler Kommentar Internationales Privatrecht, hrsg. v. Heinrich Honsell, Nedim Peter Vogt, Anton K. Schnyder und Stephen V. Berti, 3. Aufl., Basel 2013 (zit. BSK IPRG-Schnyder/Liatowitsch), Art. 86 IPRG N 17, Barbara Graham-Siegenthaler, Anhang IPR, in: Praxiskommentar Erbrecht, hrsg. v. Daniel Abt und Thomas Weibel, 3. Aufl., Basel 2015 (zit. PraxKomm-Graham-Siegenthaler), Anhang IPR N 19.

85. Vgl. Manuel Liatowitsch, Ausländische Grundstücke im internationalen und im materiellen Erbrecht der Schweiz, in: Aspekte des internationalen Immobilienrechts, hrsg. v. Helmut Heiss und Anton K. Schnyder, Zürich 2011, S. 45. 


\subsection{Alternative Anknüpfungen}

a. Das IPRG kennt als alternative Anknüpfung für die Zuständigkeit den Belegenheitsort (Art. 88 IPRG), welcher vor allem für Grundstücke verwendet wird, weil sich viele Staaten mit ausländischen Grundstücken nicht befassen, insbesondere die common law-Staaten, aber auch zum Beispiel Liechtenstein. ${ }^{86}$ Die Belegenheit kennt man in der Erbrechtsverordnung als subsidiären Anknüpfungspunkt (Art. 10 EuErbVO). Diese Bestimmung bereitet Drittstaaten wie der Schweiz Probleme, weil sie sehr weit geht: Eine Nachlassbelegenheit im Sinne von Artikel 10 EuErbVO in einem Mitgliedstaat ist dann gegeben, wenn sich (im Zeitpunkt der Entscheidung) körperliche Gegenstände (unbewegliche oder bewegliche Sachen) in einem Mitgliedstaat befinden (Belegenheit), Gegenstände oder Rechte in einem öffentlichen Register eines Mitgliedstaates verzeichnet sind (unbewegliche Sachen, Schiffe, Luftfahrzeuge oder Immaterialgüterrechte) sowie bei Forderungen gegenüber einem Schuldner in einem Mitgliedstaat. Wenn Erblasser ein Staatsangehöriger ist (lit. a) oder innerhalb der vergangenen fünf Jahre seinen gewöhnlichen Aufenthalt in diesem Mitgliedstaat gehabt hat (lit. b), ist die Zuständigkeit für den ganzen Nachlass gegeben, wenn eine solche Beziehung fehlt, wird die Zuständigkeit auf den inländischen Nachlass begrenzt (Abs. 2 EuErbVO) ${ }^{87}$ Diese Bestimmung führt dazu, dass man auch in der Schweiz wohnhaften Erblassern zur Vermeidung von Zuständigkeitskonflikten rät, sich von Vermögen in der EU zu trennen, wozu neben Unternehmen und Grundstücken auch Aktien von europäischen Gesellschaften gehören. ${ }^{88}$

b. Der IPRG knüpft alternativ am Heimatort an, und zwar wenn sich das Ausland mit dem Nachlass nicht befasst (Art. 87 Abs. 1 IPRG). Im Rahmen der Revision des IPRG bereitet vor allem die Frage, wie festgestellt werden kann, dass sich das Ausland nicht mit dem Nachlass befasst, Kopfzerbrechen. Der Vernehmlassungsentwurf von Artikel 87 Absatz 1 IPRG (VEIPRG $)^{89}$ bezweckt richtigerweise, Kompetenzkonflikte zu vermeiden. Angesichts der Tatsache, dass in der Praxis nur wenige Fälle von tatsächlichen Kompetenzkonflikten auftreten, stellt sich die Frage, ob sich die Schweiz auch dann zurückziehen soll, wenn aus-

86. Vgl. ZK-Künzle (Fn. 77), Art. 88 IPRG N 5; vor Inkrafttreten der EuErbVO galt dies auch Frankreich, Belgien, Luxemburg und Spanien, vgl. ZK-Künzle (Fn. 77), Art. 86 IPRG N 21.

87. Vgl. Anatol Dutta, Kommentar zu Art. 1-84 EuErbVO, in: Münchener Kommentar zum Bürgerlichen Gesetzbuch, Band 10: Internationales Privatrecht I, 6. A., München 2015 (zit MünchKomm IPRG I-Dutta), Art. 10 EuErbVO N $13 \mathrm{ff}$.

88. Vgl Kinga Weiss/Manuel Bigler, Die EU Erbrechtsverordnung - Neue Herausforderungen für die internationale Nachlassplanung aus Schweizer Sicht, successio 8 (2014) 178.

89. Art. 87 Abs. 1 VE-IPRG (siehe Fn. 75) lautet: 'Die betreffenden Gerichte oder Behörden können ihre Zuständigkeit zusätzlich von der Untätigkeit der Behörden eines oder mehrerer ausländischer Heimatstaaten des Erblassers, des Staates seines letzten gewählten gewöhnlichen Aufenthalts oder soweit es um einzelne Nachlasswerte geht, des Staates, in dem sie liegen, abhängig machen.' ländische Staaten extensive Zuständigkeitsregeln haben oder wenn solche Erbschaftsverfahren aus der Sicht des schweizerischen IPRG gar keine anerkennungswürdigen Entscheide bzw. Dokumente produzieren. Zudem sollte es nicht im freiem Ermessen der Erbschaftsbehörden bzw. Gerichte stehen, ob die Zuständigkeit im Einzelfall von der Untätigkeit ausländischer Behörden und Gerichte abhängig gemacht wird (Kann-Vorschrift). Schliesslich ist zu bedenken, dass in vielen Staaten keine Negativ-Bescheinigungen eingeholt werden können.

\subsection{Rechts- und Zuständigkeitswahl}

a. Sowohl im schweizerischen IPRG als auch in der europäischen EuErbVO gibt es die Möglichkeit einer Rechts- und Gerichtsstandswahl. Nach Artikel 87 Absatz 2 und Artikel 90 Absatz 2 IPRG kann der Erblasser, der sich im Ausland befindet, die (schweizerische) Heimat-Zuständigkeit bzw. das (schweizerische) Heimat-Recht für seinen Nachlass wählen. Nach Artikel 5 EuErbVO können die Erben den Gerichtsstand am (europäischen) Heimatort und nach Artikel 22 EuErbVO das (europäische) Heimatrecht wählen. Einschränkend wirkt allerdings, dass Artikel 5 EuErbVO vom Recht eines Mitgliedstaates ausgeht und somit zum Beispiel für einen Schweizer, der in den Niederlanden lebt, nicht angewendet werden kann. ${ }^{90}$

b. Doppelbürger sind im geltenden schweizerischen Recht von einer Wahl des Heimatrechts (Art. 90 Abs. 2 IPRG) ausgeschlossen, ${ }^{91}$ während Mehrstaat(l)er in der EU ein Recht wählen dürfen, dem sie zur Zeit der Rechtswahl oder im Zeitpunkt des Todes angehören (Art. 22 Abs. 1 Satz 2 EuErbVO). ${ }^{92}$ De lege ferenda wird deshalb vorgeschlagen, dass Doppelbürger auch in der Schweiz ihr ausländisches Heimatrecht wählen können (Art. 90 Abs. 2 VEIPRG). ${ }^{93}$ Gleiches soll für die Wahl der Heimatzuständigkeit gelten (Art. 86 Abs. 3 VE-IPRG). ${ }^{94}$

90. Vgl. (bezüglich Deutschland) Kurt Siehr, Deutsch-schweizerische Erbfälle nach Inkrafttreten der EuErbVO, in: Tatsachen - Verfahren - Vollstreckung, hrsg. v. Peter Breitschmid etc., Zürich 2015, S. 690.

91. Vgl. BSK IPRG-Schnyder/Liatowitsch (Fn. 84), Art. 90 IPRG N 13; Tarkan Göksu, Kommentar zu Art. 86-96 IPRG, in: Handkommentar zum Schweizer Privatrecht, 3. Aufl., Zürich 2016 (zit. CHK-GÖKSU), Art. 90 IPRG N 7.

92. Vgl. MünchKomm IPRG I-Dutta (Fn. 87), Art. 22 EuErbVO N 3; Majorie Devisme, Les conséquences pratiques du règlement (UE) $n^{\circ}$ 650/2012 du 4 juillet 2012 dans les successions franco-suisses, in: Journée de droit successoral 2015, hrsg. v. Paul-Henri Steinauer etc., Bern 2015, S. 124.

93. Der Vorschlag von Art. 90 Abs. 2 VE-IPRG (Fn. 75) lautet: 'Personen, die über eine oder mehrere ausländische Staatsangehörigkeiten verfügen, können, ungeachtet einer allfälligen schweizerischen Staatsangehörigkeit, durch letztwillige Verfügung oder Erbvertrag den Nachlass einem der betreffenden Heimatrechte unterstellen'.

94. Der Vorschlag von Art. 86 Abs. 3 VE IPRG (Fn. 75) lautet: 'Personen, die über eine oder mehrere ausländische Staatsangehörigkeiten verfügen, können, ungeachtet einer allfälligen schweizerischen Staatsangehörigkeit, durch letztwillige Verfügung oder Erbvertrag den Nachlass ganz oder teilweise der Zuständigkeit eines der betreffenden Heimatstaaten unterstellen'. 


\subsection{Erb- und Eröffnungsstatut}

Während das schweizerische Recht neben dem Erbstatut für einzelne Fragen (sichernde Massnahmen und das Nachlassverfahren) auch das Eröffnungsstatut anwendet (Art. 92 IPRG), ${ }^{95}$ gilt nach Artikel 23 EuErbVO für alle Fragen, welche von der Erbrechtsverordnung erfasst werden, das Erbstatut. Der Unterschied ist kleiner, als dies auf den ersten Blick erscheinen mag, weil das Erbverfahrensrecht von den Mitgliedstaaten der EuErbVO selbst bestimmt wird und sich nach der lex fori richtet (Eröffnungsstatut). ${ }^{96}$

\subsection{Formstatut}

a. Das für die Form letztwilliger Verfügungen massgebende Recht (Formstatut) bestimmt sich nach Artikel 93 IPRG durch das Haager Testamentsübereinkommen (HTestÜ), ${ }^{97}$ welches für die Schweiz seit dem 17.10.1971 gilt. $^{98}$ Für die Niederlande (und somit zwischen diesen beiden Staaten) gilt es seit dem 1. Juli 1982. Seit dem Inkrafttreten der Erbrechtsverordnung gilt innerhalb der Mitgliedstaaten Artikel 27 EuErbVO, welcher weitgehend identische Regeln enthält. $^{99}$

b. Im Rahmen der Revision des IPRG wird vorgeschlagen, die Regeln von Artikel 93 IPRG nach Artikel 94 Absatz 4 VE-IPRG und Artikel 95 Absatz 4 VEIPRG zu verschieben. Weil eine Änderung der Nummerierung von Bestimmungen kaum etwas bringt, aber leicht verwirren kann, ist zu hoffen, dass dieser Vorschlag nicht umgesetzt wird.

\subsection{Anerkennung ausländischer Ausweise}

a. Artikel 96 regelt, welche im Ausland ergangenen Rechtsakte eines im Ausland eröffneten Nachlasses im Inland anerkannt (und vollstreckt) werden. Man spricht in diesem Zusammenhang von indirekter Zuständigkeit der ausländischen Behörden. ${ }^{100}$ Artikel 96 ist eine lex specialis zu Artikel 25 ff. IPRG, insbesondere Artikel 26 IPRG. ${ }^{101}$ Ausländische Rechtsakte werden in der Schweiz anerkannt, wenn sie auf einem Verfahren beruhen und von einer Amtsperson erstellt wurden. ${ }^{102}$ Diesen Anforderungen genügen unter anderem die niederländische Erbrechtserklärung (verklaring van erfrecht), ${ }^{103}$ aber auch die romanischrechtlichen Notorietätsurkunden ${ }^{104}$ und selbst die
Selbstdeklarationen des italienischen (atto sostitutivo) und des anglo-amerikanischen Rechts (affidavit). ${ }^{105}$

b. Verlangt ein Erbe von einer Privatperson (Bank, Versicherung, Treuhänder und so weiter) Auskunft oder die Herausgabe eines Erbschaftsaktivums, kann diese zur Prüfung der Gültigkeit des Legitimationsausweises ein Exequaturverfahren (Art. 25 ff. IPRG) durch die zuständige Behörde ${ }^{106}$ verlangen. ${ }^{107}$ In diesem Verfahren sind ausländische Ausweise regelmässig zu beglaubigen und $\mathrm{zu}$ übersetzen. In der Bankpraxis werden übliche Legitimationspapiere ohne weiteres akzeptiert und zwar im Rahmen der Regeln, welche vom Eidgenössischen Grundbuchamt für die wichtigsten Länder (unter anderen die Niederlande) ${ }^{108}$ für den Grundbucheintrag entwickelt wurden. ${ }^{109}$

c. Die EuErbVO sieht für das Europäische Nachlasszeugnis unter den Mitgliedstaaten kein Anerkennungsverfahren vor (Art. 69 Abs. 1 EuErbVO). Wie weit sich dieses Instrument praktisch bewährt, muss sich noch zeigen, zumal es gewisse Anlaufprobleme gibt (komplizierter Antrag, zu kurze Laufzeit von 6 Monaten, unklarer Gutglaubensschutz, unklares Verhältnis zu den nationalen Erbscheinen). ${ }^{110}$ In der Schweiz ist zu erwarten, dass es ohne weiteres anerkannt wird (mit ähnlichen Wirkungen wie die schweizerische Erbbescheinigung). ${ }^{111}$
95. Zum Eröffnungsstatut vgl. ZK-Künzle (Fn. 77), Art. 92 IPRG N 23 ff.

96. Vgl. MünchKomm IPRG I-Dutta (Fn. 87), Art. 23 EuErbVO N 32 f.

97. Vgl. Übereinkommen vom 5. Oktober 1961 über das auf die Form letztwilliger Verfügungen anwendbare Recht (Haager Testamentsübereinkommen - HTestÜ - SR 0.211.312.1)

98. Zu den Vertragsstaaten vgl. www.hcch.net/en/instruments/conven tions/status-table/?cid=40

99. Vgl. MünchKomm IPRG I-Dutta (Fn. 87), Art. 27 EuErbVO N 2.

100. Vgl. BSK IPRG-Schnyder/Liatowitsch (Fn. 84), Art. 96 IPRG N 1

101. Vgl. Hans Kuhn, Anerkennung und Wirkungen ausländischer Erbausweise im schweizerischen Recht, SZIER 11 (2002) 3.

102. Vgl. Einzelrichter Kantonsgericht Zug vom 17.3.2008, ZG GVP 2008, 229, E. 7: syrischer Richter.

103. Vgl. Bundesamt für Justiz, Ausländische Erbfolgezeugnisse als Ausweis für Eintragungen im schweizerischen Grundbuch, Bern 2001, Niederlande $1 / 1$.

104. Vgl. PraxKomm-Graham-Siegenthaler (Fn. 84), Anhang IPR N 114.
105. VgI. Kuhn (Fn. 101), SZIER 11 (2002) 7

106. Vgl. Hans Rainer Künzle, Berner Kommentar zu Art. 517-518 ZGB (Die Willensvollstrecker), Bern 2011, Vorbem. zu Art. 517-518 ZGB N 113.

107. Vgl. OGer. ZH vom 2.8.2001, ZR 2002 Nr. 28: Anerkennung eines probate (Testamentseröffnungsverfügung aus Israel).

108. Es handelt sich um die Länder Dänemark, Deutschland, Frankreich, Israel, Italien, Kanada, Niederlande, Österreich, Portugal, Slowakische Republik, Südafrika, Tschechien, Ungarn, Vereinigtes Königreich und Vereinigte Staaten.

109. Vgl. Bundesamt für Justiz (Fn. 103)

110. Vgl. Michael E. Völkl, Das Europäische Nachlasszeugnis - Ein erster Überblick, AnwZert ErbR 6/2015 Anm. 2.

111. Vgl. Ivo Schwander, Die EU-Erbrechtsverordnung AJP 23 (2014) 1103. 